\title{
In vivo imaging of neurodegeneration in Caenorhabditis elegans by third harmonic generation microscopy
}

\author{
E.J. GUALDA*, G. FILIPPIDIS*, M. MARI* \\ M. VLACHOS $\dagger$, C. FOTAKIS ${ }^{*} \ddagger \&$ N. TAVERNARAKIS $†$ \\ *Institute of Electronic Structure and Laser, Foundation of Research and Technology-Hellas, 71110 \\ Heraklion, Crete, Greece \\ $\dagger$ Institute of Molecular Biology and Biotechnology, Foundation of Research and Technology, 71110 \\ Heraklion, Crete, Greece \\ $\ddagger$ Department of Physics, University of Crete, Crete, Greece
}

Key words. Caenorhabditis elegans, imaging, microscopy, neuron degeneration, THG.

\section{Summary}

In this study, neurodegeneration phenomena were investigated, by performing third harmonic generation imaging measurements on the nematode Caenorhabditis elegans, in vivo. The in vivo, precise identification of the contour of the degenerating neurons in the posterior part of the nematode and the monitoring, in real time, of the progression of degeneration in the worm, through third harmonic generation imaging measurements, were achieved. Femtosecond laser pulses $(1028 \mathrm{~nm})$ were utilized for excitation. Thus, the THG image contrast modality comprises a powerful diagnostic tool, providing valuable information and offering new insights into morphological changes and complex developmental processes in live biological specimens.

\section{Introduction}

Ageing and senescence are complex processes that dramatically impact human health. Elucidation of the basic molecular mechanisms underlying the progressive decline in cellular function that accompanies ageing and eventually leads to senescence will have an immediate impact on the design of novel interventions that could reduce or delay age-related deterioration in humans. The ageing process precipitates dramatic alterations in the physiology of all organisms, including reduced cellular function, compromised resistance to stress and pathological agents, and increased likelihood of developing age-related diseases. Among the most characteristic pathologies associated with old age are numerous late-onset neurodegenerative disorders such

Corresponding to: G. Filippidis. Tel: ++ 302810391320 ; fax: ++302810391318; e-mail: filip@iesl.forth.gr as Alzheimer's, Parkinson's and Huntington's diseases. In addition to stroke, which also inflicts loss of neuronal cells, these conditions account for ever-increasing debilitation among the elderly. Recent studies in model organisms such as the nematode Caenorhabditis elegans (C. elegans) and the fruit fly Drosophila melanogaster, which offer the prowess of sophisticated genetic approaches, have uncovered significant, novel aspects of the molecular mechanisms that underlie both neurodegeneration and the ageing process. These advances hold promise that the intimate link between the aged state and the manifestation of several neurodegenerative diseases will be deciphered. Consequently, the development of imaging techniques for the in vivo monitoring of biological activities and the detection of the changes of the internal structures during the neuronal degeneration process in model organism (such as C. elegans samples) is essential for the advancement of biomedical sciences.

Within the field of microscopy there is the need for imaging complicated structures with higher contrast and higher twoand three-dimensional spatial and temporal resolution. This need has been addressed by the use of laser scanning confocal microscopy. Confocal microscopy has been very successful due to its reliability, robustness and its ability for imaging living specimens. However, confocal microscopes can offer only few of the capabilities possible with the non-linear imaging modalities.

Non-linear imaging methodologies such as two-photon excitation fluorescence second harmonic generation (SHG) and third harmonic generation (THG) are well-established non-destructive techniques that have been recently used as tools for the in vivo imaging and mapping of sub-cellular biological structures and processes (Muller et al., 1998; Moreaux et al., 2000; Campagnola \& Loew, 2003; Mohler et al., 2003; Pons et al., 2003; Zipfel et al., 2003; Debarre 
et al., 2005; Filippidis et al., 2005). Their main advantages in comparison with the conventional one-photon confocal microscopy are the capability of intrinsic three dimensionality, the increased axial resolution, the ability to section deep within tissues (Centonze \& White, 1998) and the reduction of "out of focal plane' photobleaching and phototoxicity in the biological specimens. Additionally, the use of femtosecond ( $\left.f_{s}\right)$ lasers enables high peak powers for efficient non-linear excitation, but at low enough energies so that biological specimens are not damaged.

THG is associated to the real part of $\chi^{(3)}$ susceptibility. THG signal is enhanced when the focus is in an interface between two media with different refractive index (Squier \& Muller, 2001). Thus, THG can be used to image the different organs of a transparent biological sample with a three-dimensional microscopy capability (Chu et al., 2003; Sun et al., 2004; Debarre et al., 2006). This non-invasive, non-destructive imaging modality can be used for the imaging of structural features and molecular processes of transparent biological samples. Differences in THG coefficients at different locations in the biological sample translate to differences in THG intensities that can be measured. The non-resonant nature of THG, which provides minimum sample disturbance, renders this technique desirable for biological studies. A specific advantage of THG is that, whereas SHG requires a medium without inversion symmetry, third-order processes such as THG are allowed in any medium. As reported previously (Chu et al., 2003; Sun et al., 2004; Gualda et al., 2008), a multiphoton microscope can be easily adapted for measuring THG signals. It is important to note that the information obtained through THG imaging measurements is recorded without the need of exogenous fluorescent labels. This cannot be achieved by employing confocal microscopy. Additionally, THG microscopy provides quantitative information (e.g. for velocimetric analysis; Debarre et al., 2004) in comparison with differential interference contrast microscopy. Differential interference contrast microscopy generates a three-dimensional physical relief corresponding to the variation of optical density of the sample, emphasizing lines and edges, though providing only qualitative information and not a topographically accurate image.

In this study, we present the in vivo detailed monitoring of the progression of neurodegeneration in the posterior part of the nematode C. elegans through THG measurements. Detailed and specific structural and anatomical features have been visualized, by recording THG signals. We suggest that, THG microscopy imaging measurements comprise a valuable diagnostic tool for in vivo monitoring of neuron degeneration. Mutations in a number of nematode genes, including but not limited to mec-4, deg-1 and deg-3, lead to the death through necrosis of particular neurons in the posterior part of the worms. We have followed neurodegeneration during development in mutant animals harbouring cytotoxic mec$4(d)$ alleles using THG microscopy.

\section{Experimental set-up}

The experimental set-up is based on the one used for our previous work (Gualda et al., 2008). Modifications have been introduced in order to increase the sensitivity and the lateral resolution of the system. These are the replacement of the monochromator by an interference filter to detect all the bandwidth of the THG signal, the use of a more sensitive photomultiplier tube (PMT) with a preamplifier unit and the use of galvanometric mirrors for the sample scan. The laser used is an Amplitude Systems t-pulse laser operating at $1028 \mathrm{~nm}$ (Amplitude Systems, Pessac, France). The average power of the laser is $1 \mathrm{~W}$, with pulse duration of less than $200 \mathrm{fs}$ and a repetition rate of $50 \mathrm{MHz}$. The beam is directed to a modified optical microscope (Nikon Eclipse ME600D, Nikon Corp., Tokyo, Japan) using suitable dichroic mirrors, and is focused tightly onto the sample by an objective lens with high numerical aperture (Nikon $50 \times$ N.A. 0.8, Nikon Corp.). To ensure that the back aperture of the objective is filled a telescope system has been used. The average laser power on the specimen is $30 \mathrm{~mW}(0.6 \mathrm{~nJ}$ per pulse). No damage in the sample is observed at this power. Biological samples are placed between two round glass slides (Marienfeld 0.06-0.08 mm, Paul Marienfeld GmbH \& Co. KG, Lauda-Königshofen, Germany) at a specially design holder. A charge-coupled device (CCD) camera (PixeLINK PL A662, PixeLink, Ottawa, Canada) is used for observation. In order to get high-resolution images the scanning is performed by a pair of galvanometric mirrors (Cambridge Tech. $6210 \mathrm{H}$, Cambridge Tech., Inc., MA, USA) and the focal plane is selected with a motorized translation stage (Standa 8MT167-100, Standa, Vilnius, Lithuania). The minimum step of the stage is $1 \mu \mathrm{m}$.

For thin samples, most of THG signals propagate with the laser beam and are collected and collimated by employing a common condenser lens (Carl Zeiss Plan-Apochromat $100 \times$ N.A. 1.4 oil immersion, Carl Zeiss, Oberkochen, Germany). A dichroic mirror (Enhanced Aluminium Mirror, Thorlabs, Newton, NJ, USA) is used to reflect the transmitted beam. After passing through a 340-nm interference filter (Hoya U 340, Hoya, Long Beach, CA, USA) the signal is sent to a photomultiplier tube (Hamamatsu H930504, Hamamatsu, Japan) connected to a lock-in amplifier (SR810 Stanford Research Systems, Standford Research Systems, Inc., Sunnyvale, CA, USA). The use of a lockin amplifier provides a very good noise rejection, and measurements can be performed in less stringent lighting conditions. The synchronization of the lock-in amplifier with the galvanometric mirrors and the motorized stage is achieved through specially designed software (National Instruments, Labview 7.1, National Instruments, Austin, TX, USA). The recording time is $1 \mathrm{~ms}$ in every step. Matlab 7.1 (MathWorks, Inc., Natick, MA, USA) is used for processing of the obtained data. 


\section{Biological sample}

We have utilized the nematode worm C. elegans, which has emerged as a particularly convenient and versatile model organism, for our analysis. The adult C. elegans hermaphrodite is about $1 \mathrm{~mm}$ long and $80 \mu \mathrm{m}$ in diameter. Animals can be grown on a diet of Escherichia coli, on agar plates or in liquid medium. The transparency of the animal body allows easy visualization and monitoring of cellular processes and has permitted recording and determination of the complete pattern of cell divisions that generate the 959 somatic cells of the adult. Despite its apparent simplicity, there is a high degree of differentiation; worms have muscle cells, hypodermis, a nervous system, intestine, gonads, glands and an excretory system. Moreover, the complete wiring diagram of its 302 neurons and their connecting synapses has been elucidated from serial section electron micrographs. Furthermore, the transparency of the worm allows appropriate fluorescent markers to be readily followed in vivo. In addition, the detailed description of the worm nervous system provides a unique setting for studying the action of many drugs that act on the central nervous system in humans. Given that essential neuronal processes and functions (e.g. neurotransmitters and their neuronal receptors) are highly conserved between $C$. elegans and vertebrates, such endeavours are expected to yield information relevant to humans. The strains used in this study were the following: wild-type N2 Bristol isolate, deg-3(u662)V, mec-4(u231)X, N2;Ex[ $\left.\mathrm{p}_{m e c-4} \mathrm{GFP}\right]$ and mec4(u231)X;Ex[ $\left.\mathrm{p}_{m e c-4} \mathrm{GFP}\right]$. We followed standard procedures for C. elegans strain maintenance, crosses and other genetic manipulations (Brenner, 1974). Nematode cultivation temperature was kept at $20^{\circ} \mathrm{C}$. Gravid adult animals were allowed to lay eggs overnight and eggs were collected and allowed to hatch within a time window of $4 \mathrm{~h}$. Before each experiment, early larvae the L1 stage of development were collected and anaesthetized by immersing to $0.5 \mathrm{M}$ of sodium azide $\left(\mathrm{NaN}_{3}\right)$, and subsequently mounted on glass slides.

\section{Results}

Swelling deaths induced by gain-of-function mutations in genes of the degenerin family, such as mec- 4 , deg- 1 and mec10, exhibit morphological features of necrotic cell death. Such deaths are distinct from programmed cell death in several ways: Cells undergoing programmed cell death, appear to be compacted and 'button-like', whereas cells undergoing degenerative cell death appear to be swollen and enlarged (Fig. 1). In addition, distinct ultrastructural changes accompany the two types of death, programmed cell deaths transpire within the hour, whereas degenerative deaths occur over several hours. The time of onset of degenerative death correlates with initiation of degenerin gene expression, and the rapidity with which death occurs correlates with dose of the toxic allele. These observations are consistent with the hypothesis that a threshold ion influx is needed to initiate the degenerative process. Ultrastructural analysis has established that degeneration initiates with striking infoldings of the plasma membrane. Small tightly wrapped membranous whorls are the first indications of pathology. Subsequently, internalized whorls grow in size and large vacuoles appear. Cell body volume can increase 100-fold during this process. The nucleus becomes distorted and chromatin aggregates. Internal degradation of cell contents then follows shortly (Troulinaki \& Tavernarakis, 2005).

In order to evaluate the usefulness of THG signals for the in vivo imaging of degenerating neurons in the posterior part of C. elegans, a three-dimensional scan has been performed. In Fig. 2, nine slices are shown, measured at different depths in the area of the tail of transgenic larvae. The scanning area is $15 \times 15 \mu^{2}$ with a resolution of $300 \times 300$ points. The distance between adjacent slices is $1 \mu \mathrm{m}$. The precise morphology of the degenerating neuron it is easily recognizable, due to the collection of strong THG signals generated at the membrane. Furthermore, high intensity THG signals are detected from various structures with optical discontinuity, such as sub-cellular organelles, inclusion, cavities and lipid depositions, in the C. elegans posterior body segment (Debarre et al., 2006; Gualda et al., 2008). In addition, THG signals arising from the contour of the posterior part of the larva are recorded.

To demonstrate the potential of THG as a diagnostic tool for neurodegeneration studies, the evolution of morphological alterations in degenerating neurons of $C$. elegans larvae was tracked over extended periods of time. In Fig. 3 can be seen the changes suffered from a dying neuron over $2 \mathrm{~h}$. The scanning area is $15 \times 15 \mu \mathrm{m}^{2}$ with a resolution of $300 \times 300$ points. The

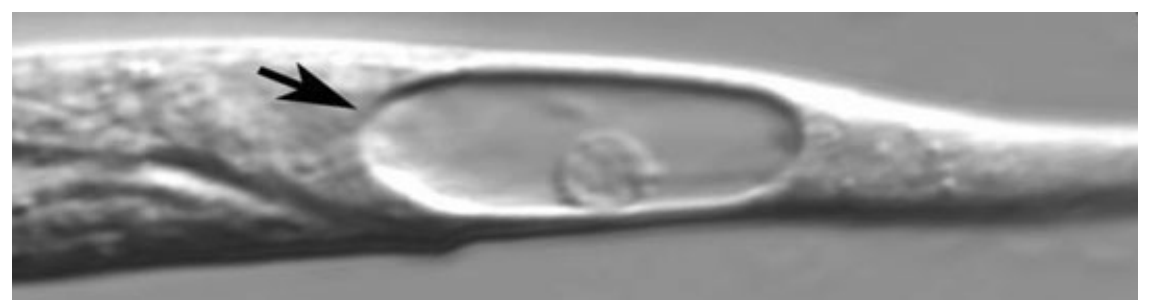

Fig. 1. Degenerating neuron in the posterior part of a C. elegans larva. The image was obtained by employing a differential interference contrast optical microscope (Nomarski, DIC). 

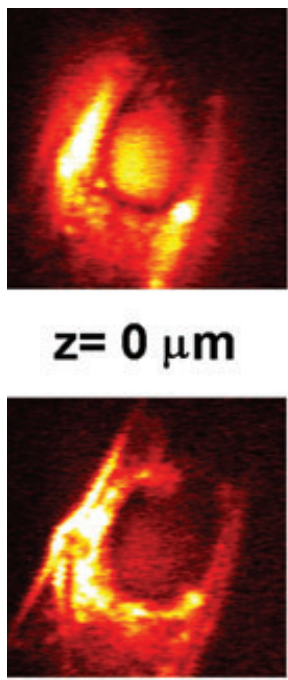

$z=3 \mu \mathrm{m}$

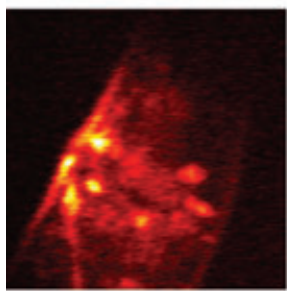

$z=6 \mu \mathrm{m}$

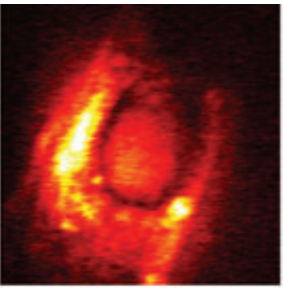

$z=1 \mu \mathrm{m}$

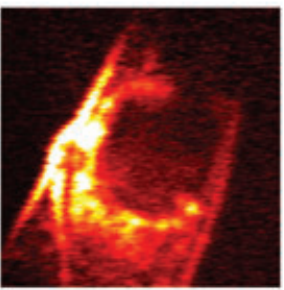

$z=4 \mu \mathrm{m}$

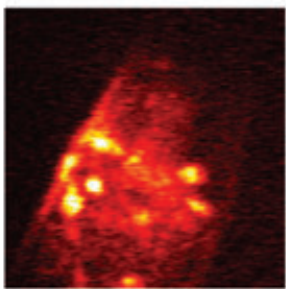

$z=7 \mu \mathrm{m}$

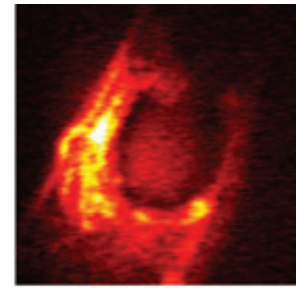

$z=2 \mu \mathrm{m}$

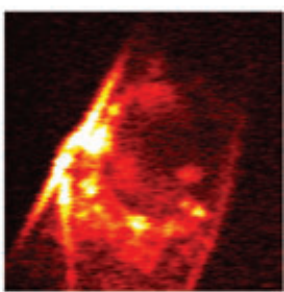

$z=5 \mu m$

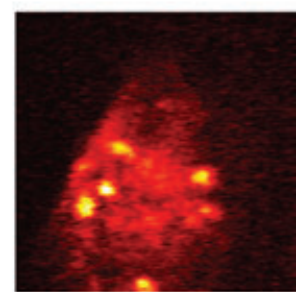

$z=8 \mu \mathrm{m}$

Fig. 2. THG three-dimensional scanning of the posterior part of a transgenic C. elegans larva. The separation between slices is $1 \mu \mathrm{m}$ and the scanned area is $15 \times 15 \mu \mathrm{m}^{2}$.

(a)

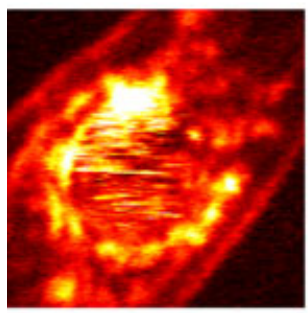

0 hours (b)

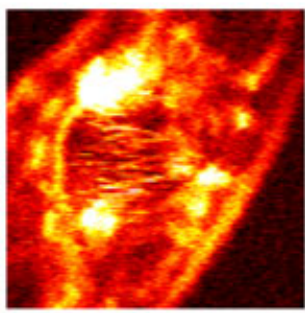

1 hour (c)

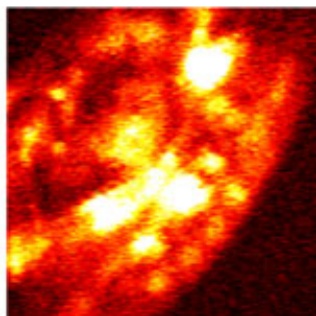

2 hours

Fig. 3. THG signal from the posterior part of a transgenic C. elegans larva. The time lapse between images is $1 \mathrm{~h}$ and the scanned area is $15 \times 15 \mu \mathrm{m}^{2}$.

specimens were kept in darkness at intervals between the THG measurements in order to minimize unwanted photodamage effects. The first image (Fig. 3a) corresponds to the initial observation of the biological sample (time zero) and the neuron presents a great degree of degeneration. After $1 \mathrm{~h}$ (Fig. $3 \mathrm{~b}$ ), a reduction of the volume of the swollen, degenerating neuron is apparent. This effect becomes more obvious with time (Fig. 3c, after $2 \mathrm{~h}$ ). An elongation of the neuron is also observed. In Fig. 3a and b, the THG signal arising from the sub-cellular organelles (e.g. nucleus) inside the neuron cell is detectable. Consequently, THG imaging measurements permit not only the detection of structural features but also the identification of biological processes occurring in vivo in degenerating neurons. In Fig. 3(c) (2 h after), the elimination 

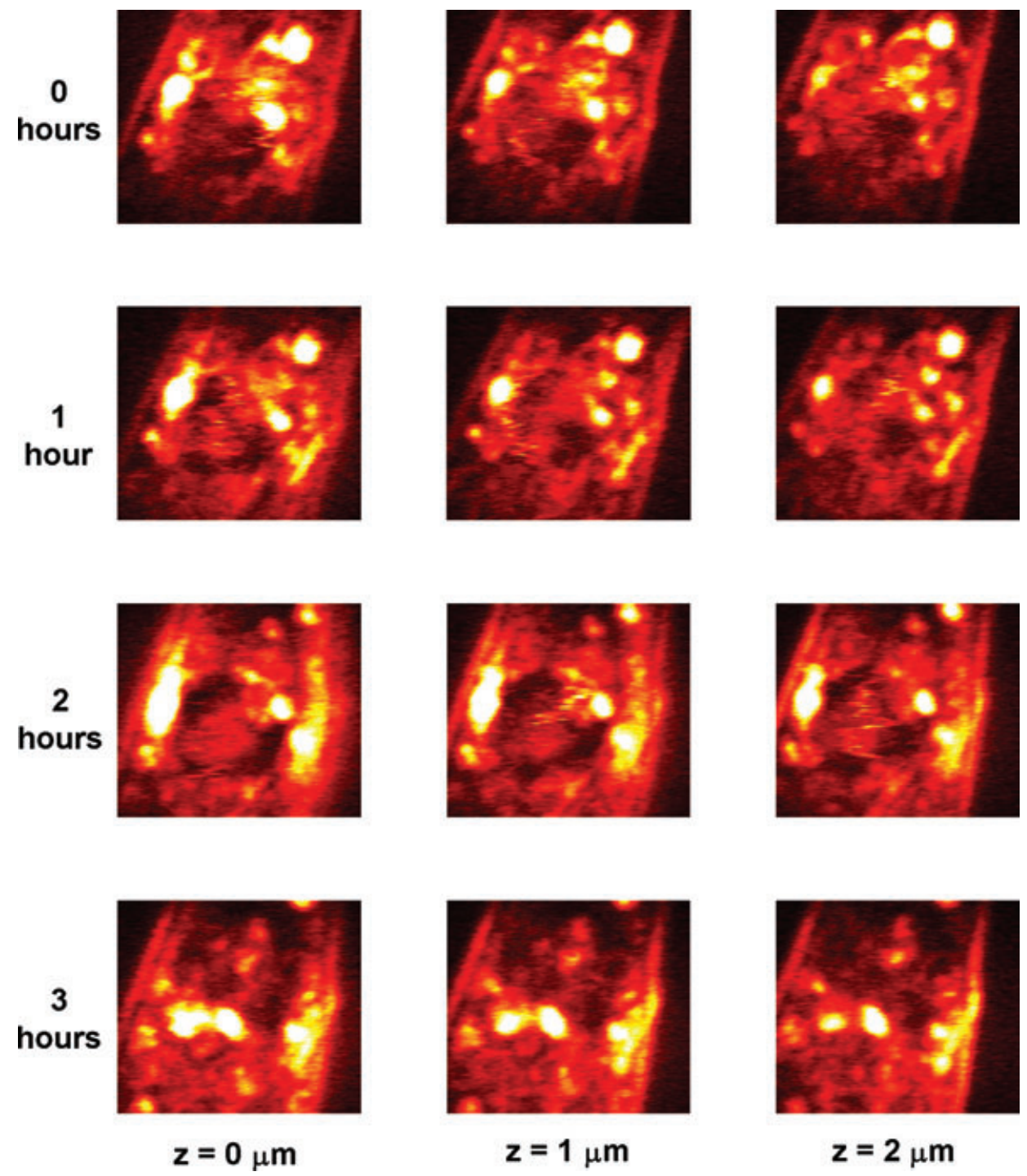

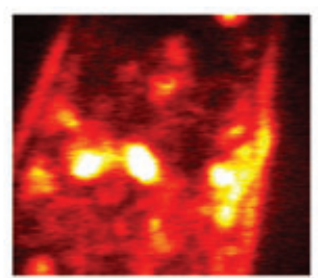

$z=1 \mu \mathrm{m}$

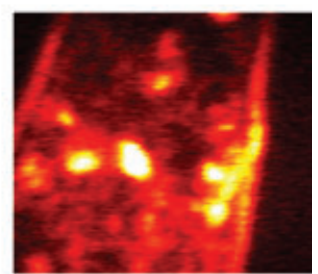

$\mathrm{z}=\mathbf{2} \mu \mathrm{m}$

Fig. 4. THG signal from the posterior part of a transgenic C. elegans larva. The time lapse between images appears in the vertical axis and the depth of scanning in the horizontal. The scanned area is $15 \times 15 \mu \mathrm{m}^{2}$.

of the THG signal arising from the inner fast moving organelles inside the cell, can be observed. We note that, more than 20 different samples were irradiated and the results obtained were repeatable. The presented THG images (Fig. 3) comprise the most representative data, typical of the evolution of morphological alterations in a degenerating neuron of $C$. elegans larvae.

Finally, in order to exclude the possibility that the detected morphological changes in the degenerating neurons of the worms are due to movement of the sample, different sets of three-dimensional measurements have been performed with a time lapse of $1 \mathrm{~h}$ between them (Fig. 4). To avoid the possible sample drift, nematodes were imaged by measuring 10 sequentially stacks and afterwards selecting the stacks that correspond to the same sample plane. Some anatomical features, such us the anus and lipid bodies have been used as a reference. For these series of measurements 10 different samples were tested and the results are in perfect agreement with the previous measurements (one image per hour at a certain depth). All sets of measurements and the obtained results lead us to the conclusion that the detectable morphological changes are not due to sample drift.

Figure 4 depicts the time progression of the three sections, where the neuron vacuole was initially optimally observed. The scanning area is $15 \times 15 \mu \mathrm{m}^{2}$ with a resolution of $300 \times 300$ points. Two hours after commencing observation the neuron increases in volume, presenting a rapid collapsing during the next hour and almost disappearing. This series of morphological alterations can be followed not only by analysing the neural membrane perimeter, but also by the 
thorough observation of the movement of the surrounding cells and lipid depositions in the tail of the animals, as the neuron grows or diminishes. Moreover, after $3 \mathrm{~h}$, no internal organelles are detectable and the degradation of the neuronal cell is obvious. These data suggest that degenerative neuron death occurs over a period of several hours. These observations, obtained by performing THG measurements, are in perfect agreement with other studies of neurodegeneration in C. elegans (Troulinaki \& Tavernarakis, 2005; Syntichaki et al., 2007).

\section{Conclusions}

THG imaging has proved to be a very useful, non-destructive diagnostic tool for monitoring cellular processes in vivo. The recorded highly reliable results, in a number of specimens obtained during the course of the current study, proved the potential of THG imaging modality to provide information related to a specific problem with high biological importance (neuronal degeneration). We suggest that this non-linear technique may be used in tandem with other optical techniques, such as differential interference contrast modality, in order to obtained complementary structural information from various biological samples. The main advantages of using endogenous higher harmonic generation, (such as THG) as microscopic contrast mechanism for in vivo biological studies, are that no fluorescence is required and the common issues of photodamage, phototoxicity, photobleaching, dye availability or dye toxicity can be eliminated. Moreover, in vivo cellular processes can be monitored with high spatial and axial resolution continuously for a long period of time, because the unwanted interactions (e.g. thermal, mechanical side effects) are minimized.

We anticipate that, this non-linear imaging approach comprises a useful tool for studying cell-fate determination at early developmental stages and it can be useful for the extraction of valuable quantitative volumetric and structural information from diverse cell types and model organisms.

\section{Acknowledgements}

This work was supported by the UV Laser Facility operating at IESL-FORTH under the European Commission 'Improving Human Research Potential' program (RII3-CT2003-506350) and by the Marie Curie Transfer of Knowledge project 'NOLIMBA' (MTKD-CT-2005-029194).

\section{References}

Brenner, S. (1974) The genetics of Caenorhabditis elegans. Genetics 77, 71-94.
Campagnola, P.J. \& Loew, L.M. (2003) Second-harmonic imaging microscopy for visualizing biomolecular arrays in cells, tissues and organisms. Nat. Biotechnol. 21, 1356-1360.

Centonze, V.E. \& White, J.G. (1998) Multiphoton excitation provides optical sections from deeper within scattering specimens than confocal imaging. Biophys. J. 75, 2015-2024.

Chu, S.W., Chen, S.Y., Tsai, T.H., Liu, T.M., Lin, C.Y., Tsai, H.J. \& Sun, C.K. (2003) In vivo developmental biology study using noninvasive multi-harmonic generation microscopy. Opt. Express 11, 30933099.

Debarre, D., Supatto, W., Farge, E., Moulia, B., Schanne-Klein, M.C. \& Beaurepaire, E. (2004) Velocimetric third-harmonic generation microscopy: micrometer-scale quantification of morphogenetic movements in unstained embryos. Opt. Lett. 29, 2881-2883.

Debarre, D., Supatto, W. \& Beaurepaire, E. (2005) Structure sensitivity in third-harmonic generation microscopy. Opt. Lett. 30, 21342136.

Debarre, D., Supatto, W., Pena, A.M. et al. (2006) Imaging lipid bodies in cells and tissues using third-harmonic generation microscopy. Nat. Methods 3, 47-53.

Filippidis, G., Kouloumentas, C., Voglis, G., Zacharopoulou, F., Papazoglou, T.G. \& Tavernarakis, N. (2005) Imaging of Caenorhabditis elegans neurons by second harmonic generation and two-photon excitation fluorescence. J. of Biomed. Opt. 10, 024015.

Gualda, E.J., Filippidis, G., Voglis, G., Mari, M., Fotakis, C. \& Tavernarakis, N. (2008) In vivo imaging of cellular structures in Caenorhabditis elegans by combined TPEF, SHG and THG microscopy. J. Microsc. 229, 141150 .

Mohler, W., Millard, A.C. \& Campagnola, P.J. (2003) Second harmonic generation imaging of endogenous structural proteins. Methods 29, 97-109.

Moreaux, L., Sandre, O., Blanchard-Desce, M. \& Mertz, J. (2000) Membrane imaging by simultaneous second-harmonic and two-photon microscopy. Optics Lett. 25, 320-322.

Muller, M., Squier, J., Wilson, K.R. \& Brakenhoff, G.J. (1998) 3D microscopy of transparent objects using third-harmonic generation. J. Microsc. 191, 266-274.

Pons, T., Moreaux, L., Mongin, O., Blanchard-Desce, M. \& Mertz, J. (2003) Mechanisms of membrane potential sensing with second-harmonic generation microscopy. J. Biomed. Optics 8, 428-431.

Squier, J. \& Muller, M. (2001) High resolution nonlinear microscopy: a review of sources and methods for achieving optimal imaging. Rev. Sci. Instrum. 72, 2855-2867.

Sun, C.K., Chu, S.W., Chen, S.Y., Tsai, T.H., Liu, T.M., Lin, C.Y. \& Tsai, H.J. (2004) Higher harmonic generation microscopy for developmental biology. J. Struct. Biol. 147, 19-30.

Syntichaki, P., Troulinaki, K. \& Tavernarakis, N. (2007) eIF4E function in somatic cells modulates ageing in Caenorhabditis elegans. Nature 445 , 922-926.

Troulinaki, K. \& Tavernarakis, N. (2005) Neurodegenerative conditions associated with ageing: a molecular interplay? Mech. Ageing Dev. 126, 23-33.

Zipfel, W.R., Williams, R.M. \& Webb, W.W. (2003) Nonlinear magic: multiphoton microscopy in the biosciences. Nature Biotec. 21, 13691377. 